\title{
Reading the Signs: Intersemioticity and Non-Verbal Communication
}

\section{Introduction}

Jakobson (1971) stressed the semiotic value of all five senses in human society such that syncretic messages, that is, those based on a combination of several sign patterns are brought together. The reading and interpretation of non-verbal signs has often involved a comparison with verbal communication in order to examine the extent to which the two sign systems, that is 'body language' and spoken language, are congruent. The study of non-verbal communication in terms of posture, gestures, and facial expressions has long tradition in terms of a populist psychological literature that is predicated upon the notion that the body can speak louder than words, that it can reveal 'hidden' thoughts and feelings. This offering, of a revelatory analysis of the body's sign value, is a defining rhetorical aspect of this field of study. The power to reveal the body's language trades upon a linkage between body and mind in which the embodied person emits signals that displays their inner psychology. These signals are taken as requiring expert interpretation in terms of reading their intersemiotic value and meaning. Analyses of this kind trade upon forging a linkage between body and mind in which the embodied person emits signals that displays their "inner" psychology during interaction and particularly in relation to status, power and relationships. This sustains a visual ideology of the communicative body.

The study of non-verbal communication or body language as it is commonly known, is popular amongst laypeople as well as being an established area of academic psychology. There has been a thriving populist and academic literature in the field in recent years (e.g. Beattie 2003; Eaves and Leathers 2017; Hall and Knapp 2009; Hall, Horgan, 
and Murphy 2019; Hinde 2005; Matsumoto, Frank and Hwang 2013; Wharton 2009). There is also a huge applied side to this work in terms of coaching programmes associated with interaction in the world of business and personal relationships (e.g. Dunbar and Bernhold 2019; Collett 2004; Glass 2012; Pease and Pease 2011; Wezowski and Wezowski 2012). Much of the appeal of this area rests upon the idea that body language involves people communicating their 'true' thoughts and feelings. Work in this area has traded upon Birdwhistle's (1970: 427) findings that around two thirds of emotional content is communicated by non-verbal cues. It is for this reason that there is an obvious appeal in reading these signals and de-coding what they mean. The revelatory power of being able to de-code these signals provides much of the appeal and rhetorical power of this kind of study.

Bodies are viewed as communicating socially shared 'meanings' such as joy, interest, boredom, status etc., some of which are unconscious whilst other are taken as being controllable. The idea of the body as the site of a struggle for communicative intent has a resonance with Goffman's (1959) work on self-presentation but less attention has been paid to the underlying process of intersemiotic translation involved. This in itself is a powerful rhetoric and in the case of non-verbal communication the notion of direct visual 'experience' that requires translation is used as the bedrock for interpreting what a person is thinking or feeling. The body is positioned as a communicative device in which every movement is taken as displaying some meaning or insight into what someone is saying or doing.

\section{Movements and Minds}

In the study of non-verbal communication there is one major underlying assumption: signs that require translation. What is said, in what way and with what accompanying body movements, are taken as requiring interpretation in order to assess the degree of congruence between them and what they reveal about the person during the interaction. There is an assumed intra-psychic world that is mediated through the outer world of the body which is in turn is relayed to other minds. The nuances of body movements in terms of facial expressions and bodily positions and gestures are taken as being the focus of investigation. Therefore, the body in this view is treated as a window onto something else; an inner world that requires to be exposed. 
It is this revelatory discourse that gives the study of body language so much of its rhetorical power. To be able to read the mind of another by analysing the micro-movements of their body is an area that has captured the public imagination and shows no sign of abating. It is perhaps no accident that this should be the case given that the mass appeal of this area of study has been applied to topics such as personal relationships, politics and business. The ability to communicate effectively and to be influence and 'read' others is often crucial in these aspects of people's lives. It is also the case that these are just the areas where success in doing so carries much in terms of individual 'rewards'.

Viewing the body as a means of communication in which there is an exchange of signs about some 'inner' psychological state sets up the problem of interpretation and of interactional 'rules' and norms. It raises such issues as: How can we know the mind of others by watching them? What is the status of these bodily signals with respect to what a person is saying about something? Which movements and actions are intentional and which are unconscious? Can we be trained to control these signals to some degree? Which signals convey the most important information about what a person feels? Which signals are best related to what a person thinks? All of these questions flow from this communication model of nonverbal behaviour in terms of an assumed transmission of 'information' as a basis for inter-subjectivity. This assumed perceptual-cognitive basis for interaction is, of course, the basis of much psychological investigation which trades on a the assumption that people are concerned with seeing and interacting with one another in order to understand what they are thinking and feeling. This is part of a wider cultural commonplace, the notion of an 'inner/outer' dualism, and one in which the disciplines of psychology and sociology have played a considerable part in actively maintaining. Moreover, it provides a means of trading on notions of 'sense making' as well as the portrayal of people's 'inner' psychological states.

Cognition is regarded as the element of control and providing a basis for thinking before acting. The affective or emotional element is taken being spontaneous and representing 'feelings' and it is this aspect that is often taken as being a major part of what body language can reveal. The emotional state of a person as displayed through non-verbal cues is often taken as a reliable indicator of a person's 'true' feelings. The physiology of these bodily movements and facial expressions is regarded as virtually beyond the total control of the individual. Nevertheless, there is a vast industry based upon trying to literally discipline the body to bring these under conscious rational control: a case of mind over body. It is also interesting to note that much of the discourse on the study of body 
language revolves around the detection of deception by reading nonverbal leakage, or in Goffman's (1959) terms, the signals that are given off.

This way of viewing these signals is based on the notion of what people say as largely intentional and based upon 'thoughts', 'ideas' and 'views' where these are taken as being the result of some process of reasoning. Emotion provides a means of supporting this process in terms of action or as something that skews or bypasses the reasoning process and is ultimately manifested in the body. This duality is interesting in terms of the ways in which emotion can be presented and interpreted as a means of characterising action. As Edwards (1997) notes emotions are a very flexible accounting resource whereby they can be contrasted with cognitions in terms of their less deliberative nature, taken as being as 'understandable' and appropriate as how any reasonable person would react, characterised as being the outcome of events or in the nature of the person, treated as being kept under the control of a person's reasoning or as reactions that resist control, presented as the interaction of mental and physiological systems, as natural, or as derived from moral and ethical concerns.

These discursive resources can also be applied to the visual domain when people index emotions to how various bodily aspects look or are occluded from view. The visibility of 'emotions' as indexed to the body is therefore a major cultural resource and means of referring to issues of accountability. Studying participants' orientations to the visibility of emotions, either in terms of direct psychological accounting, or in terms of orientating towards aspects of the inner/outer dualism allows for a level of analysis in term of the study of the orderliness of social action. In this way a major cultural dualism is maintained: taking people's 'outward' non-verbal signs as representations that can be translated into cultural texts in terms of what they are like 'inside'. This derives from accountability within practices rather than as the result of some sort of inner mental cognitive processing and exchange of representations.

This model is orientated to as part of the social practices that people engage in and they ways in which they index psychological states to visible aspects of the body. It is something that people orientate to in terms of a communicative translation process that reveals an individual's attitudes, beliefs, motives, goals, judgements etc. In effect, this cultural orientation is one of a treating the body as a window onto an intrapsychic world as something that is normatively attended to as a means of accomplishing order within social practices. The nature of this order is founded upon an orientation of participants employing a discourse related to mental processes in order to account for how they perceive matters and 
as the basis for action. In this way bodily signals are placed prior to this operation, as having happened and that need to be 'understood' within interaction.

It is this intersemiotic model therefore that drives the 'expertise' of those in the study of non-verbal behaviour. The stories of emotional and other states laid upon the body through notions of revealing the signals that is given off leads to a discourse of functionality as related to particular bodily 'channels': vocal quality, facial movements, eye movements; posture, orientation, distance and touch, gestures, appearance, and even chemical. These channels are then related to functions such as marking identity and status; the display of emotional states; role relationships; joint focus of attention; rituals, and illustrators. There is also a move to consider the inter-dependence of verbal and non-verbal communication in terms of such aspects as being able to do two different things at once (e.g. the regulation of conversation through eye contact; the use of pointing in giving verbal directions) or the use of as both channels in affording faster transmission.

\section{Viewing the Body as Communicating an Inner Psychology}

Jakobson (1971) suggested that intersemiotic translation or transmutation involves the interpretation of the signs of a sign system with the signs of another sign system. He also saw the translation process as involving two processes that take place simultaneously: recoding and transposing. However, the distinction between the changing and retaining processes sits within a psychological view of translation (see Deely 2004). To this Jakobson stresses "all five external senses carry semiotic functions in human society" (1971: 701) thereby adding a cultural dimension to his thought. He also stressed the importance of distinguishing between homogeneous messages based upon a single sign system and syncretic messages involving the combination of multiple sign systems (1971: 705).

What is interesting about Jakobson's view of intersemiotic translation is the extent to which auto-communicative activity underwrites culture. Culture is in a permanent process of intersemiotic translation and the interpretation of non-verbal signs actualises the flow of cultural communication and it relationship with language Ruthrof (2000). Gorlée (1989) takes up this focus on the active and cultural nature of intersemiotic translation through a consideration of Wittgenstein's later philosophy 
(Philosophical Investigations (PI)1953). She draws attention to Wittgenstein's notion of language games as a form of life and that he underlined that "the term language-game is meant to bring into prominence the fact that the speaking of language is part of an activity" (PI: §133). These instances of "operating with words", have a built-in nonverbal component and are bound up with forms of life within cultures. Wittgenstein also argues that 'we follow rules blindly' (PI: §219), in the sense that we proceed in an unthinking way that requires no intermediary step such as interpretation. One way that we may do this is through engaging in the language game of translating the body as representing an inner psychological world, of solving the 'problem' of hearing language being spoken and seeing the speaker's non-verbal movements as part of the communication process. An example that captures this well the gesture of a father 'showing' a teenage daughter what to pick up in order to clean up her room (Gerhardt 2019). This type of nonverbal communication, as well as being a specific activity, is also part of an encounter where that response acts as a powerful means of displaying, not only frustration or annoyance, but also local roles and moral obligations. It is part of a language game enacted between a parent and a reluctant child in which 'showing' what do to by picking up an object to be tidied away has a much greater directive force than simply pointing to it.

This language game involves treating the person as a psychological agent in terms of 'mental processes' being required to operate upon how they view others in the interactional world in order to 'make sense' of them. In this way the bodily signs are placed prior to this operation, as having happened and needing to be 'understood'.

In this process of communication there is a realm of embodied interaction and a realm of assumed mental operations requiring to be brought together in order to apprehend or grasp the nature of these non-verbal signals. The selection and active constitution of these signals as a social practice is occluded through the reification of 'reality' and 'mind', that requires to 'understood' or 'made sense' of by an inner mental processing system that 'perceives' that outer reality.

This association between the labelling of 'perceptual moments' in the understanding of body language and the mental operations that have been applied to them provides for a means of establishing a rationalist account of non-verbal communication in terms of functionality. The body must be attended to in terms of discrete signals and in this way a perceptual-cognitivist form of viewing the body is actively maintained. It is this outer body that is taken as presenting itself as requiring 'interpretation' or 'understanding' in terms of an active 'inner' response. 
It can also be the basis for creating a version of temporality in which what 'has happened' is taken as being apparent in the person's actions. The body is therefore divided into expressive functions that require interpretation as 'language'.

This can be found at 'lay' and 'expert' levels of analysis. At the lay level of analysis this is considered as an common aspect of everyday intersemiotic translation as people go about their lives. Thus Berger (2014: 23) writes:

We often "watch" people when we sit in cafes or restaurants but we also scrutinize them when we look at the facial expressions of our wives, of our husbands and of our children, of actors and actresses in plays, films and television programs, and of politicians. We consider things such as hair styles, hair colors, eye colors, body structure, body language, styles of eyeglasses, brands of smart phones, purses, clothes, watches, smart phones, and so on [...] ad infinitum.

Berger further goes on to note the 'expert' interest in this area by drawing attention to the work of the psychologist Paul Ekman in arguing that "[...] facial expression provides information about our emotions and moods, reflects cognitive activity such as boredom and perplexity, can reveal truthfulness and lying, and can offer information about mania, schizophrenia and depression" (2014: 23).

Therefore the ubiquitous nature of observing body language as a form of communication, both at lay and expert levels, give credence to the distinction between the two. People may in their day-today conduct engage in reading body language but this is at the level of everyday moral encounters and self-presentation (Goffman 1959). On the other hand, there is a justification for expert analysis of body language, including facial expressions, as a means of information about mental states that may, in some cases, point to medical conditions. These different circumstances can be considered as different language games, one rooted in interpersonal moral issues, the other in diagnostic evaluation. However, there are occasions when expert and lay concerns are involved in the same language game, and this is often related to 'reading' people more effectively for the purpose of business or personal relationships. As previously noted, the authority of experts resides in their assumed enhanced ability to decode body language in ways that others may quite literally not see.

Whether lay or expert, one of the most important features of body language is emotional expression. This is commonly conceived of as related to various emotional clusters. For example, in a review of the 
importance of research in this area Kaushal (2013: 16-17) draws attention to the following:

Aggressive: tightened jaws, stiff facial muscles, a sombre face and straight staring eyes, thumping the table with tight fists to make a point, raised voice, animated gestures with overuse of arms and fingers - and sometimes of the whole body.

Submissive: head held down, closed stance, nods agreement much more frequently than desired, chin kept down, palm open to indicate this openness.

Attentive: gazing of the eyes towards the speaker, mild nods of heads at periodic intervals, saying ' $\mathrm{hmmm}$ ', 'yes', 'is it so', at appropriate points.

Nervous: frequent (visible) swallowing of the saliva, unsteady voice often accompanied by a stammer, avoiding eye contact, and head bent down.

Upset: tightened jaws, deliberate frown on the forehead, taking off the reading glasses, eyes closed occasionally, avoiding eye contact with others or unstable or losing control over emotions (e.g., voice shaking, out-of-control gestures).

Bored: prolonged or frequent yawns or eyes shut for a duration, blank stares when eyes are open.

Relaxed: head straight, chest upright and balanced body, no folds or frown on the forehead, easy and quite breathing, gentle smile on face and calm yes.

Power: common body language gestures that indicate power are sitting at the head of a table, standing/sitting from an elevated platform, thumping fist on the table, or accompanied by key aides at all times etc.

Defensive: tightly folded arms, crossed legs, sitting on a reverse chair or showing nervousness while giving excuses etc.

What is striking about these kinds of descriptors is their apparent banality. They appear commonsensical and somewhat obvious. However, this apparent obviousness betrays our ability to engage in a language game of intersemiotic translation in which we are able match up these terms with bodily 'signals'. Insofar we are familiar with this language game it is because we have learned it as part of our culture through being socialized into it. This socialization process does not necessarily consist of matching people's outward body language to some inner notion of their mental state, nor does it entail a process of 'interpretation' on the part of the observer. Such attempts abstracting, regularizing and generalizing this process is what gives authority to the apparent expertise of those who decode these signals. 
The availability of a shared visual experience is built into and presupposed by this intersemiotic translation process in order to then correspond with the kinds of emotion descriptors outlined above. The capacity to use observations of other people's body movements and facial expressions in order to identify, recognize and describe these as signals and to relate them to language, is something that is therefore part of the enculturation and socialization process. Observing others in this sense is not simply observing in general but rather is related to particular circumstances and various language games. These might, for example, involve engaging in acceptances and refusals, giving views and opinions, maintaining institutional role-appropriate behaviour, displaying affection, annoyance, anger and so on. Learning to engage in these activities through either attempting to control one's own body language or observing that of others is not an abstract process of inner interpretation. On the contrary, it is all about participation in activities that are not separable from learning the public nature of what kinds of bodily signals are taken as mattering in terms of their relationship with the nature of the concepts that we learn.

Therefore, in short, it is the fact that we have learned to engage in this translation process as a matter of course that enables us to treat attempts to enhance its revelatory power as being something that we can connect with. However, such attempts tend to formalize what we already 'know' and in so doing glosses over these in an abstract way as 'body language' that speaks of an inner psychology. The popularity of this kind of approach derives from the idea of making the hidden visible through the interpretation of this body language. However, this only 'works' so long as an inner/outer dualism is maintained as a channel through which signals can move and be interpreted. By considering the relationship between the visual observation of the body in interaction and the language games that they sit within, then it is possible to adopt a more reflexive stance on the power of the discourse of 'body language' and its appeal as giving the power to see the body and through it.

\section{Conclusion}

The construction of an 'inner/outer' dualism in people's embodied interaction presents a world of texts of 'meaning' in which a process of intersemiotic translation is brought to bear upon viewing the others in order to 'make sense' of them, and in terms of inner 'thoughts' and 'feelings'. In this way the inner/outer dualism is maintained as a pervasive 
discursive cultural common place: the construction of 'mind' as an active perceptual-cognitive system 'working on' perceptual signals.

Such accounting is a matter of public practice as people engage in various forms of social relations that are mediated through different social and organisational practices. The basis for a person's agency has to be intelligible and therefore such accounts must attend to this in their construction. In this sense the person-as-viewer, as positioned as 'outside' of another external psychological agent and who must in the course of the account employ his or her own inner thought processes in order to know the other's mind. Perhaps this is what makes the languagegame of non-verbal communication such a powerful one; a discourse of perceptual-cognitivism in which the positioning of interlocutors is based upon the achievement of displaying and interpreting certain kinds nonverbal signals that may or may not be congruent with verbal interaction. The complexity of these signals, and being able to read them correctly, is taken as being required to 'understand' others. However, this can be considered as an attempt to relate various semi-conscious aspects of the body or automatisms with a rational discourse of the performative body and associated emotion-based categories. In so doing it represents a means of defining and rationalising the unruliness of the body, a means of subjecting it to an abstract language game of intersemiotic translation.

\section{References}

Beattie, Geoffrey (2003). Visible Thought: The New Psychology of Body Language. New York: Routledge.

Berger, Arthur Asa (2014). "Semiotics and Society". Society 51 (1): 22-26.

Birdwhistell, Ray L. (1970). Kinesics and Context: Essays on Body Motion Communication. Oxford, UK: Ballantine.

Collett, Peter (2004). The Book of Tells: How to Read People's Minds from Their Actions. London: Bantam Books.

Deely, John (2004). "The Intersemiosis of Perception and Understanding". The American Journal of Semiotics 20 (1/4): 211-253.

Dunbar, Norah E., and Quinten Bernhold (2019). "Power and Nonverbal Behavior". In: Power in Close Relationships. Eds. Christopher R. Agnew and Jennifer J. Harman. Cambridge: Cambridge University Press.

Eaves, Michael, and Dale G. Leathers (2017). Successful Nonverbal Communication: Principles and Applications. London: Routledge.

Edwards, Derek (1997). Discourse and Cognition. London: Sage. 
Gerhardt, Cornelia (2019). "Showing'as a Means of Engaging a Reluctant Participant into a Joint Activity". In: Elisabeth Reber and Cornelia Gerhardt. Embodied Activities in Face-to-face and Mediated Settings: Social Encounters in Time and Space. (137-175). Cham, Switzerland: Springer (Palgrave Macmillan). Glass, Lillian (2012). The Body Language Advantage: Maximize Your Personal and Professional Relationships with This Ultimate Photo Guide to Deciphering. Beverly, MA: Fair Winds.

Goffman, Erving (1959). The Presentation of Self in Everyday Life. New York: Doubleday.

Gorlée, Dinda L. (1989). “Wittgenstein, Translation, and Semiotics”. Target 1 (1): 69-94.

Hall, Judith A., Terrence G. Horgan, and Nora A. Murphy (2019). “Nonverbal Communication". Annual Review of Psychology 70: 271-294.

Hinde, Robert A. (ed.) (1975). Non-verbal Communication. Cambridge: Cambridge University Press.

Jakobson, Roman (1971). "Language in Relation to Other Communication Systems". In: Roman Jakobson. Selected Writings 2. Word and Language. (697708). The Hague: Mouton.

Kaushal, Saurabh (2013). "Contribution of Non Verbal Language in Communication: A Study of Non-Verbal Communication". Asian J. of Adv. Basic Sci. 2 (1): 15-21.

Knapp, Mark, Judith Hall, and Terrence Horgan (2012). Nonverbal Communication in Human Interaction. ( $8^{\text {th }}$ ed.) Boston: Cengage Learning.

Matsumoto, David, Mark G. Frank, and Hyi Sung Hwang (eds.) (2012). Nonverbal Communication: Science and Applications. Los Angeles: Sage.

Pease, Allan, and Barbara Pease (2011). Body Language in the Workplace. Hachette, UK.

Ruthrof, Horst (2000). The Body in Language. London: Cassell.

Wezowski, Patryk, and Kasia Wezowski (2012). The Micro Expressions Book for Business: How to Read Facial Expressions for More Effective Negotiations, Sales and Recruitment. New Vision.

Wharton, Tim (2009). Pragmatics and Nonverbal Communication. Cambridge: Cambridge University Press.

Wittgenstein, Ludwig (1953). Philosophical Investigations. Oxford: Blackwell. 